\title{
Photometric study of selected X-ray binaries
}

\author{
T. İçli, D. Koçak and K. Yakut \\ Department of Astronomy and Space Sciences, University of Ege, 35100, \\ Bornova-İzmir, Turkey, (E-mail: icli.tugce@gmail.com)
}

Received: November 7, 2019; Accepted: January 17, 2020

\begin{abstract}
We present results of a long-term photometric multicolor optical monitoring project of selected low-mass and high-mass X-ray binaries carried out at the TÜBİTAK National Observatory (TUG). New long-term $V R I$ multicolor observations of three selected X-ray binaries with neutron star components (HZ Her, ScoX-1, SAX J2103.5+4545) were observed between 2015 and 2019 with the TUG $60-\mathrm{cm}$ telescope. The light variations of the systems are presented and discussed.
\end{abstract}

Key words: binaries: X-ray - neutron stars

\section{Introduction}

Flux variations of X-ray binaries consisting of an evolved component and a compact object (neutron star/black hole) can be observed both at X-ray and optical wavelengths. In these binary systems, the optical component usually fills its Roche lobe. Long-term light variations of both low-mass (LMXB) and highmass (HMXB) X-ray binaries provide information on astrophysical processes, particularly on hot stellar winds of the companion star, stellar activity, mass transfer between components, non-conservative mass loss and angular momentum loss from the system. In this context, long-term multi-color optical changes of selected low and high mass X-ray binaries with neutron star components, previously discussed and catalogued by İçli \& Yakut (2015), İçli (2016), are presented in this study.

Within the context of our observational project, eight X-ray binaries with neutron star components (HZ Her, Sco X-1, PSR J1023+0038, X Per, BQ Cam, V934 Her, SAX J2103.5+4545, XTE J1946+274) were examined. Some parameters of the selected systems are summarized in Table 1. Here we present longterm observational results for HZ Her, Sco X-1, and SAX J2103.5 + 4545 and we obtain a new period for the SAX J2103.5+4545 system from our new observations.

Sco X-1, which is known as the brightest LMXB, was discovered by Giacconi et al. (1962). Its optical component is V818 Sco (Sandage et al., 1966). The system has an orbital period of 0.78 days and consists of a $1.4 \mathrm{M}_{\odot}$ neutron star and a $0.42 \mathrm{M}_{\odot}$ optical component (Steeghs and Casares, 2002). Based on optical observations, the spectral type is thought to be earlier than G5. The distance 
of the system is $2.8 \pm 0.3 \mathrm{kpc}$ (Bradshaw et al., 1999) and the system exhibits a high and low state (Bandyopadhyay et al., 1999).

Discovered by observations of the UHURU satellite in 1972 (Tananbaum et al., 1972), Her X-1 (HZ Her, 4U 1656+35) is classified as an eclipsing LMXB system with an A7 spectral type (Middleditch \& Nelson, 1976; Leahy \& Scott, 1998, İcli et al., 2019). The system has an orbital period of 1.7 days and consists of an accreting neutron star with a mass of $1.5 \mathrm{M}_{\odot}$ and an optical component with a mass of $2.2 \mathrm{M}_{\odot}$ (Reynolds et al., 1997; Leahy \& Abdallah, 2014, İçli et al., 2019). Its distance is $6.6 \mathrm{kpc}$ (Reynolds et al., 1997). The system has been observed at different wavelengths (optical, ultraviolet, radio and X-ray bands), see, e.g., Shakura et al. (1997); Cherepashchuk et al. (1974); Simon et al. (2002); İçli \& Yakut (2015); İçli (2016). The X-ray flux exhibits a 35-day period variation related to the accretion disc (Scott et al., 2000; Leahy \& Abdallah, 2014; Postnov et al., 2013).

Another system within the scope of this study is SAX J2103.5+4545, discovered in 1997 with BeppoSAX. It pulsates with a period of $358.61 \mathrm{~s}$ (Hulleman et al.,1998). The system is composed of a $20 \mathrm{M}_{\odot}$ optical component with a B0 Ve spectral type (Reig et al., 2010). The orbital period of the binary system is 12.6 days (Baykal et al., 2007).

\section{New observations}

New observations were made between February 2015 and July 2019, with varying exposure times between 5, 20 and 60 seconds. Multicolour (VRI) light curves were obtained with the $60-\mathrm{cm}$ robotic telescope at the TÜBITAK National Observatory (TUG). Three systems were observed, HZ Her, ScoX-1, and SAX J2103.5+4545. Their respective periods are 1.7, 0.78, 12.6 days, and their $V$ band brightness lies between 12.5 and 13.8 magnitudes. Observations were handled with the standard difference photometry method in each observation term. IRAF/PHOT and AstroImageJ (Collins et al., 2017) were used in the reduction stage. The frame reduction was performed by subtracting the bias and dark frames and dividing by flat-field frames. Following the time correction, we performed differential photometry as we did in our prevous studies (İcli et al., 2013, Çokluk, et al., 2019, Koçak et al. 2019). AAVSO-135, AAVSO-132, AAVSO50(1) (for HZ Her), AAVSO-115, AAVSO-126, AAVSO-113 (for ScoX-1) and ID-72,131,136 (for SAX J2103.5+4545 (Reig \& Fabregat, 2015) were chosen as comparison stars. Light variations of the systems are plotted in Fig. 1.

\section{Discussion and conclusions}

X-ray binary systems consist of an early- or a late-type star and a black hole or neutron star. The presence of an accretion disk, mass loss from the system and stellar activity on the companion star affect the multi-wavelength light curves 

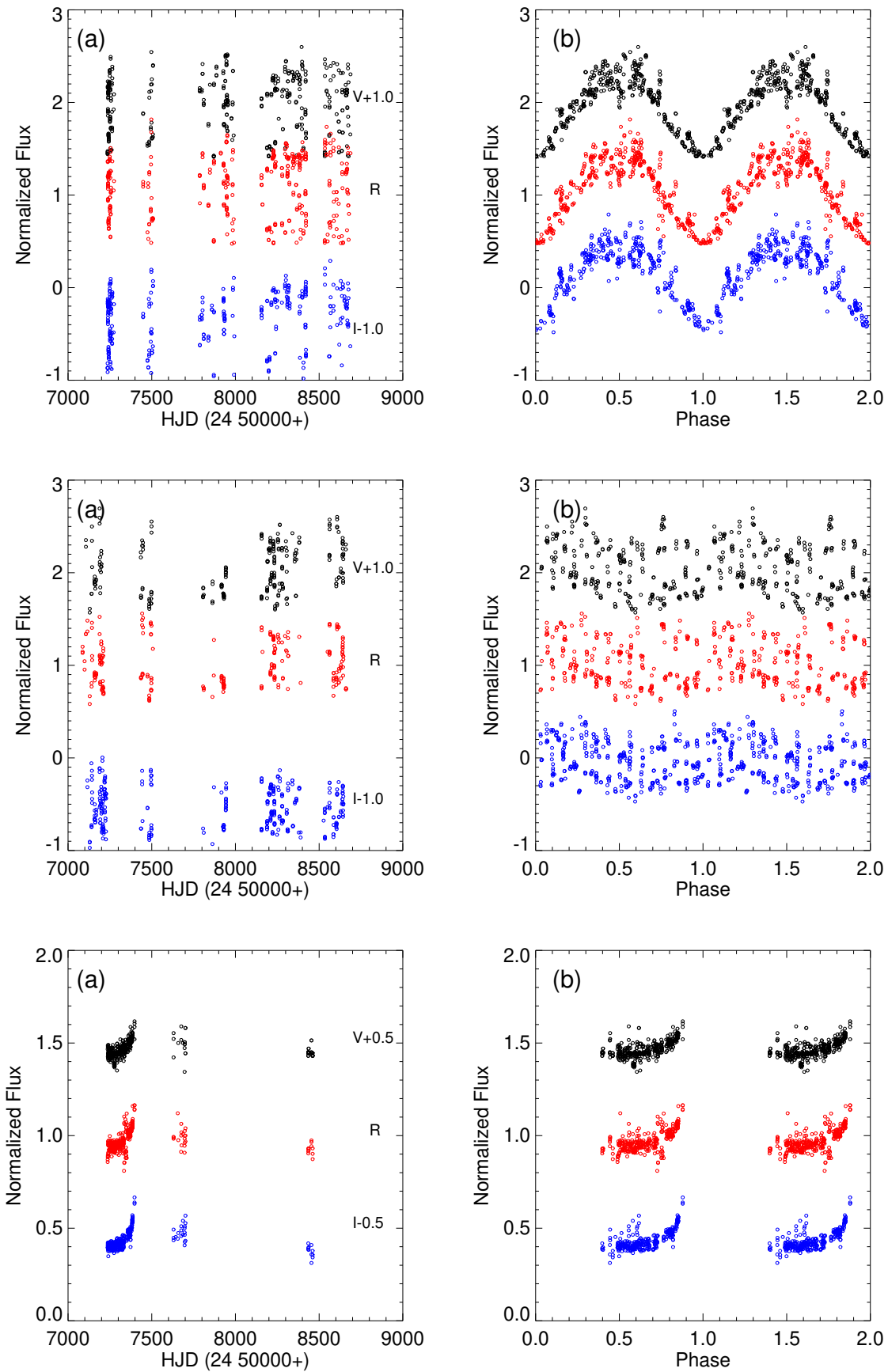

Figure 1. Long-term $V R I$ light variations of the systems in the time (a) and phase (b) domain for HZ Her (top), Sco X-1 (middle), and SAX J2103.5+4545 (bottom). 
Table 1. Parameters of selected X-ray binaries

\begin{tabular}{|c|c|c|c|c|c|c|c|c|}
\hline System & Alias & Type & $\alpha$ & $\delta$ & $V$ (mag) & $P_{\text {orb }}(\mathrm{d})$ & OTime & $\mathrm{N}_{\mathrm{obs}}$ \\
\hline$\overline{\text { PSR J1023+0038 }}$ & AY Sex & LMXB & 102348 & +003841 & 17.5 & 0.198 & $7731-8447$ & 337 \\
\hline Sco X-1 & V818 Sco & LMXB & 161943 & -153908 & 12.5 & 0.787 & $7106-8644$ & 847 \\
\hline Her X-1 & HZ Her & LMXB & 165749 & +352033 & 13.6 & 1.7 & $7236-8687$ & 1145 \\
\hline SAX J2103.5+4545 & & HMXB & 210255 & +454325 & 14.2 & 12.6 & $7236-8461$ & 1029 \\
\hline V0332+52 & BQ Cam & HMXB & 033460 & +531023 & 15.4 & 34.67 & $7059-8759$ & 2126 \\
\hline XTE J1946+274 & & HMXB & 194535 & +272043 & 16.9 & 169.2 & $7271-8659$ & 466 \\
\hline 3A $0352+309$ & X Per & HMXB & 035536 & +310025 & 6.7 & 250.3 & $7360-8461$ & 620 \\
\hline $4 \mathrm{U} 1700+24$ & V934 Her & LMXB & 170635 & +235819 & 7.6 & 4391 & $7137-8461$ & 1230 \\
\hline
\end{tabular}

of the binary systems. We obtained multicolor $(V R I)$ observations of X-ray binaries with neutron star components between 2015 and 2019 with the TUG T60 telescope. Periodic and non-periodic changes have been observed in these systems. For HZ Her, the amplitude of the light variation in the $V$ band is 1.5 mag. The middle panel of Fig. 1 shows the multicolor optical light variation of V818 Sco. The light curves of the system show amplitude variability in the $V R I$ bands. Orbital light curves of the V818 Sco (Fig. 1, middle-right panel) can differ significantly from the mean orbital light variation $(\sim 35 \%)$. Based on observations obtained over a span of five years, a period of 412 days for the HMXB SAX J2103.5+4545 was determined using the Period04 (Lenz \& Breger 2005) software. A non-periodic period variation seems to be present in the long-term observations.

Acknowledgements. We are very grateful to an anonymous referee for comments and suggestions. This study is supported by the Turkish Scientific and Research Council (TÜBİTAK - 117F188). We thank TÜBİTAK for partial support in using the T60 telescope with project numbers 15AT60-776 and 18AT160-1298. Tİ thanks TÜBİTAK for his Fellowship (2211-C). KY would like to acknowledge the contribution of COST (European Cooperation in Science and Technology) Action CA15117 and CA16104.

\section{References}

Bandyopadhyay, R. M., Shahbaz, T., Charles, P. A., et al. 1999, Mon. Not. R. Astron. Soc., 306, 417

Baykal, A., Inam, S. Ç., Stark, M. J., et al. 2007, Mon. Not. R. Astron. Soc., 374, 1108

Bradshaw, C. F., Fomalont, E. B., \& Geldzahler, B. J. 1999, Astrophys. J., Lett., 512, L121

Cherepashchuk, A. M., Kovalenko, V. M., Kovalenko, O. N., et al. 1974, Peremennye Zvezdy, 19, 305

Collins, K. A., Kielkopf, J. F., Stassun, K. G., et al. 2017, Astron. J., 153, 77

Çokluk K. A., Koçak D., Içli T., Karaköse S., Üstündağ S., Yakut K., 2019, MNRAS, 488,4520

Giacconi, R., Gursky, H., Paolini, F. R., et al. 1962, Phys. Rev. Lett., 9, 439 
Hulleman, F., in 't Zand, J. J. M., \& Heise, J. 1998, Astron. Astrophys., 337, L25

İçli, T., 2016, MSc Thesis, Binary systems with neutron star components, University of Ege

İçli, T., \& Yakut, K. 2015, The Extremes of Black Hole Accretion, 89

İçli T., Koçak D., Yakut K., 2019, IAUS, 239, IAUS..346

İçli T., Koçak D., Boz G. Ç., Yakut K., 2013, AJ, 145, 127

Koçak D., İçli T., Yakut K., 2019, IAUS, 252, IAUS..346

Leahy, D. A., \& Abdallah, M. H. 2014, Astrophys. J., 793, 79

Leahy, D. A., \& Scott, D. M. 1998, Astrophys. J., Lett., 503, L63

Middleditch, J., \& Nelson, J. 1976, Astrophys. J., 208, 567

Postnov, K., Shakura, N., Staubert, R., et al. 2013, Mon. Not. R. Astron. Soc., 435, 1147

Reig, P., Słowikowska, A., Zezas, A., et al. 2010, Mon. Not. R. Astron. Soc., 401, 55

Reig, P., \& Fabregat, J. 2015, Astron. Astrophys., 574, A33

Reynolds, A. P., Quaintrell, H., Still, M. D., et al. 1997, Mon. Not. R. Astron. Soc., 288, 43

Sandage, A., Osmer, P., Giacconi, R., et al. 1966, Astrophys. J., 146, 316

Scott, D. M., Leahy, D. A., \& Wilson, R. B. 2000, Astrophys. J., 539, 392

Shakura, N. I., Smirnov, A. V., \& Ketsaris, N. A. 1997, IAU Colloq. 163: Accretion Phenomena and Related Outflows, 379

Šimon, V., Kroll, P., Neugebauer, P., et al. 2002, NewA, 7, 349

Steeghs, D., \& Casares, J. 2002, Astrophys. J., 568, 273

Tananbaum, H., Gursky, H., Kellogg, E. M., et al. 1972, Astrophys. J., Lett., 174, L143 